\title{
Phase Transformations and Surface/Interface Properties in Functional Perovskites with Aberration-Corrected STEM/EELS
}

\author{
Jae H. Jang, ${ }^{1}$ Rohan Mishra, ${ }^{2,1}$ Young-Min Kim, ${ }^{3}$ Qian He, ${ }^{1}$ Jaekwang Lee, ${ }^{4}$ Mina Yoon, ${ }^{4}$ S.T. Pantelides, ${ }^{2,1}$ A.Y . \\ Borisevich $^{1}$ \\ ${ }^{1}$ Materials Sciences and Technology Division, Oak Ridge National Laboratory, Oak Ridge, TN 37831 \\ ${ }^{2}$ Department of Physics and Astronomy, Vanderbilt University, Nashville, TN \\ ${ }^{3}$ Korea Basic Science Institute, 169-148 Gwahak-ro, Yuseong-gu, Daejeon, 305-806, Korea \\ ${ }^{4}$ The Center for Nanophase Materials Science, Oak Ridge National Laboratory, Oak Ridge, TN 37831
}

Perovskite oxides exhibit a wide variety of electronic, magnetic, and ionic properties that make them invaluable for multiple applications in energy generation and storage. Local and global changes in oxygen stoichiometry often play a crucial role in determining these properties. For materials such as solid oxide fuel cell (SOFC) anodes and photocatalysts oxygen dynamics in the bulk and on the surface, respectively, forms the basis of their functionality. To fully understand the underlying mechanisms it is therefore necessary to characterize oxygen distribution and motion in conjunction with local electronic and magnetic properties at atomic scale. Aberration corrected STEM and EELS provide unprecedented spatial resolution for structural and chemical studies of these systems. Comprehensive image analysis via local crystallography approach enables tracking atomic column positions with picometer scale precision. In system with linear chemical expansion, local crystallography can be used to monitor oxygen content in each atomic column from interatomic spacings in HAADF images [1].

The ability to obtain chemical information from images, which can be acquired much faster than spectra, enables dynamic studies of oxygen motion with atomic resolution. For $\mathrm{LaCoO}_{3-\Omega}$ thin films, electron beam induces a series of phase transformations as the oxygen deficiency grows, mirroring bulk behavior [2]. However, in addition to observing the overall phase evolution, atomically resolved dynamic data allows us to track transformation pathways and even metastable intermediate states (Fig.1). Configuration analysis from large dynamic datasets poses substantial challenges, best solved via big data approaches [3]. The detected configurations are then used as an input for Nudged Elastic Band Density Functional Theory (DFT) calculations, revealing energy landscape of the system. Such data can be crucial for optimizing low-temperature performance of the SOFC devices.

Surfaces and interfaces of oxides can manifest properties not observed in the bulk material, enabling new functionalities. In a model vacancy ordered SOFC material lanthanum strontium cobaltite (LSCO) tetrahedrally and octahedrally coordinated Co ions alternate in the bulk, although valence state modulations are not present due to compensation by itinerant electrons due to metallic conductivity of the material. However, in the proximity of an insulating substrate, such as lanthanum strontium aluminum tantalate (LSAT) or neodymium gallate (NGO), tetrahedral and octahedral layers have distinct electronic and magnetic behavior (Fig.2). For LSCO/LSAT films, the cobalt layer adjacent to the interface is always octahedral, but for LSCO/NGO films an abundance of antiphase boundaries creates a patchwork of Co ion coordinations and therefore electronic and magnetic properties, suggesting that interface ionic conductivity for these structures may show response to electric and magnetic fields. Oxide surfaces can be studied by STEM/EELS in cross-section, enabling characterization of the surface states responsible for photocatalysis. In a model $\left(\mathrm{LaFeO}_{3}\right)_{8} /(\mathrm{Sr} \mathrm{FeO})_{1}$ perovskite digital superlattice, EELS studies indicated that, unlike the octahedral bulk, Fe at the surface is tetrahedrally coordinated. DFT calculations confirm structural observations and also suggests that, while the bulk of the superlattice is insulating and prefers G-type antiferromagnetic spin arrangement, for the surface it is energetically favorable to adopt ferromagnetic order and metallic conductivity. The effect of these phenomena on surface reactivity will be discussed, as well as the prospects of tuning this reactivity with electric and magnetic fields.[4] 
[1] Y.-M. Kim et al., Nature Mater. 11, 888-894 (2012)

[2] Hansteen et al., J. Mater. Chem. 8, 2081-88 (1998)

[3] O.S. Ovchinnikov et al., these proceedings.

[4] The research is sponsored in part by the Division of Materials Sciences and Engineering, Office of Basic Energy Sciences, U.S. Department of Energy. JL and MY were supported by the Center for Nanophase Materials Sciences which is sponsored at Oak Ridge National Laboratory by the Office of Science, Basic Energy Sciences, U.S. Department of Energy.
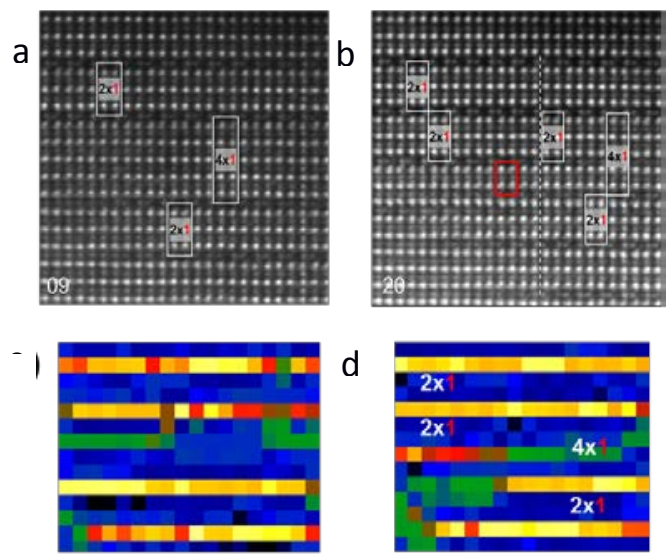

e
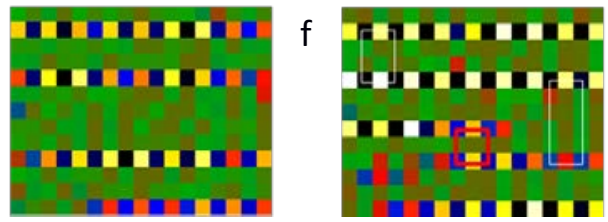

Figure 1. (a), (b) HAADF images and the corresponding (c), (d) lattice spacing maps and (e), (f) Co shift maps showing different stages of phase transformation in LaCoO3 thin films. Red boxes on Fig. 1(f) indicate metastable configurations.

a

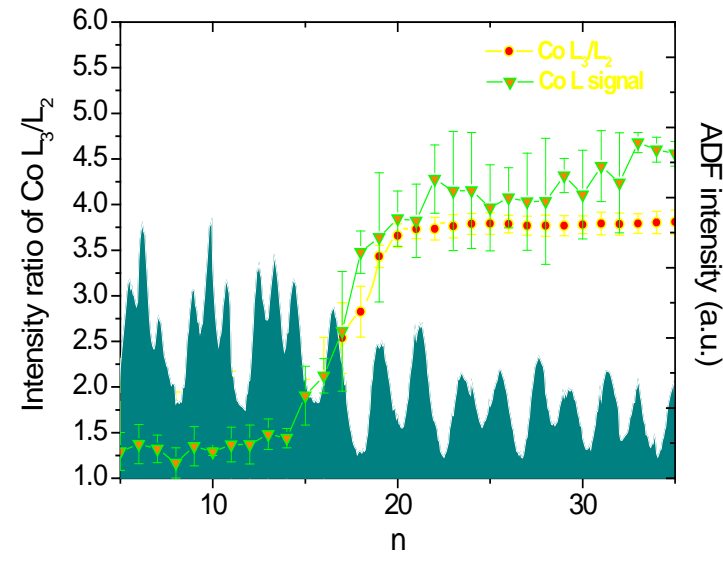

b

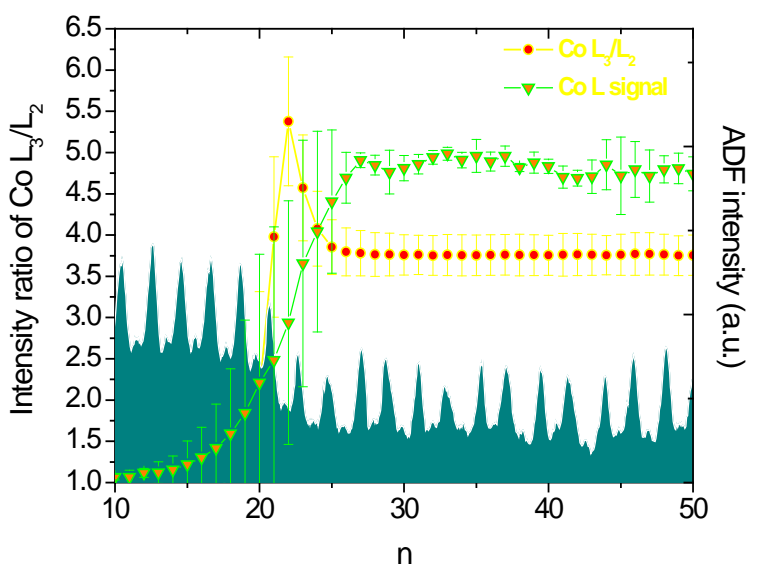

Figure 2. HAADF images and overlaid HAADF, Co L integrated signal, and CoL3/L2 ratio profiles for (a) LSCO/LSAT thin film and (b) LSCO/NGO thin film. The L3/L2 ratio anomaly in (b) is indicative of the tetrahedrally coordinated cobalt in the vicinity of the interface. 